\title{
Electromagnetic characterization of the 990 ton gapless magnets for the OPERA experiment
}

\author{
A. Cazes, ${ }^{a}$ A. Cecchetti, ${ }^{a}$ B. Dulach, ${ }^{a}$ F. lungo, ${ }^{a}$ M. Incurvati, ${ }^{a}$ D. Orecchini, ${ }^{a}$ \\ G. Peiro, ${ }^{b}$ C. Sanelli, ${ }^{a}$ F. Terranova ${ }^{a *}$ and M. Ventura ${ }^{a}$ \\ ${ }^{a}$ Laboratori Nazionali di Frascati dell'INFN, \\ Frascati (RM), Italy \\ ${ }^{b}$ CERN, Geneve, Switzerland \\ E-mail: francesco.terranova@cern.ch
}

\begin{abstract}
The instrumented targets of the OPERA neutrino experiment are complemented by two massive spectrometers based on gapless iron magnets. In 2006, a systematic assessment of their electromagnetic properties have been carried out. In this document, we report the results of such characterization and demonstrate that the achieved performance fulfill the physics requirements for the study of $v_{\mu} \rightarrow v_{\tau}$ oscillations.
\end{abstract}

KEYWORDS: Large detector systems for particle and astroparticle physics; Spectrometers; Detector alignment and calibration methods (lasers, sources, particle-beams).

${ }^{*}$ Corresponding author. 


\section{Contents}

1. Introduction 1

2. Characterization of the steels 2

3. Electric characterization of the load

4. Measurement of the magnetic field 6

5. Conclusions 9

\section{Introduction}

OPERA [1] is a long-baseline neutrino experiment located at the underground Gran Sasso laboratories; it exploits a $v$ beam (CNGS) whose energy is beyond the threshold for $\tau$ production and, therefore, it is aimed at direct observation of $v_{\mu} \rightarrow v_{\tau}$ oscillations through the reconstruction of $v_{\tau}$ charged-current (CC) interactions. The tau lepton is identified by nuclear emulsions interleaved with lead sheets ("bricks"). The neutrino target is made up of $\sim 210^{5}$ bricks; segmented plastic scintillators have been installed among the bricks to provide real-time information on the interaction vertex. In OPERA, the active mass is divided into two separate targets, each followed by a magnetic spectrometer [2]. The latter (figure 1] is able to determine the charge and momentum of penetrating tracks from their deflection (measured by chambers of drift tubes [3]) inside two iron arms; hence it contributes to suppress charm background in the $\tau$ appearance analysis and completes the kinematic reconstruction of the $v_{\mu} \mathrm{CC}$ and $v_{\tau}$ CC events with $\tau \rightarrow \mu$ decay. Each arm is made up of twelve steel layers $(5 \mathrm{~cm}$ thickness) interleaved with $2 \mathrm{~cm}$ gaps where bakelite Resistive Plate Chambers (RPC) have been installed. The arms are connected by two return yokes where copper coils are wound (20 turns per return yoke). The RPC provide track reconstruction for particles stopping in the iron bulk and a calorimetric measurement of the hadronic component.

The OPERA instrumented magnets were switched on for the first time in March 2006 and, since then, a systematic assessment of their electromagnetic properties has been carried out and is reported in this paper.

At low energy, momentum resolution for muons crossing both arms is limited by multiple scattering (MS) in iron and it is of the order of $\Delta p / p \simeq 15 \%$. At larger momenta, it is dominated by the precision of the drift-tubes and $\Delta p / p \simeq 20 \%$ at $40 \mathrm{GeV} / \mathrm{c}$. Therefore, systematic uncertainties in the knowledge of the magnetic field should be kept at a level negligible with respect to the MS-saturated resolution. Moreover, if this requirement is fulfilled, charge reconstruction efficiency exceeds $99.5 \%$ in the energy range of interest for the muonic decays of tau at CNGS (1-30 GeV) [1]]. Local B-field maps are obtained solving numerically the Poisson equation for the present geometry. Reliable results can be obtained if the non-linear response of the steel to the magnetomotive 

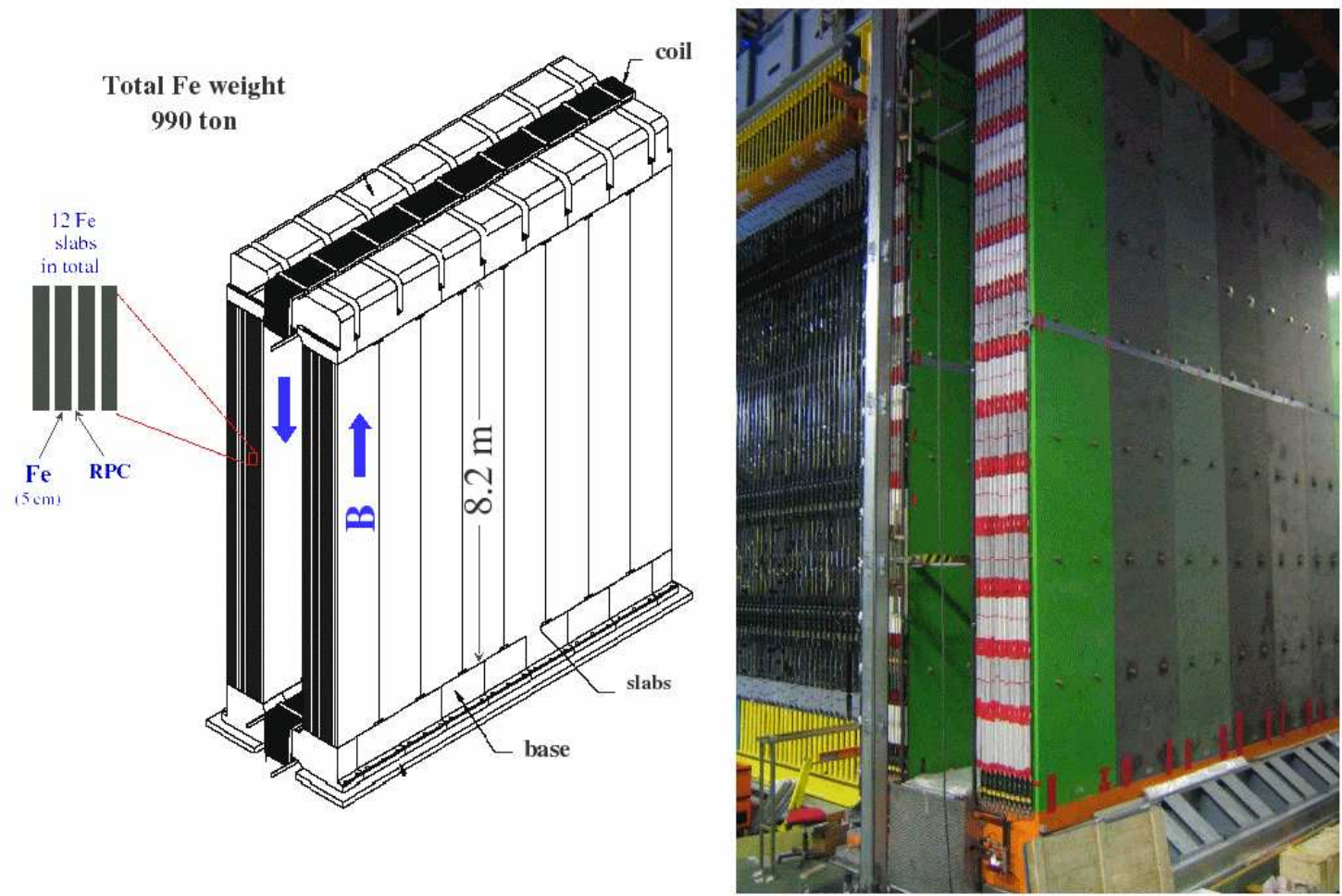

Figure 1. Schematics (left) and photograph (right) of one of the two instrumented magnets for OPERA.

forces (B-H curves) is experimentally determined: indeed, a full chemical and magnetic characterization of the steels produced for OPERA has been carried out and it is described in section 2 . Numerical expectations can be directly checked measuring the average field along a set of pickup coils positioned in the fiducial volume of the spectrometer. For the present geometry, the B-field remains constant along the arms within $4 \%$. Hence, interpolation among the measurements done at the pickup coils provides a solid test of the numerical expectations. In the following, we show that discrepancies never exceeds 5\% and, therefore, the momentum and charge resolution of the spectrometers are unaffected by the uncertainty in the determination of the field. In particular, the measurement of the electric properties of the coil and the reactive/inductive response of the magnet are described in section 3, while the determination of the magnetic field in the bulk of the iron and in air, and comparison with finite-element calculations are presented in section 7

\section{Characterization of the steels}

In OPERA, the magnets and particularly the long arms act both as subdetectors and as the basic support structure of the whole experiment. During the mounting of the target the mechanical structure of the spectrometers subdues significant stresses and, after the completion of the installation, the magnet upholds the weight of the target, the magnetic forces and possible seismic stresses. Hence, 


\begin{tabular}{|l|c|}
\hline Mechanical spec. & Value requested \\
\hline Min breaking strength & $340 \mathrm{~N} / \mathrm{mm}^{2}$ \\
Min yield strength & $225 \mathrm{~N} / \mathrm{mm}^{2}$ \\
Elongation & $>25 \%$ \\
\hline Geometrical spec. & Tolerance \\
\hline Slab height & $0.1 \mathrm{~mm}$ \\
Planarity of the slab & $3.0 \mathrm{~mm}$ \\
Orthogonality of the $1250 \mathrm{~mm}$-side w.r.t. vertical axis & $0.1 \mathrm{~mm}$ \\
Parallelism between the top and bottom $1250 \mathrm{~mm}$-side & $0.1 \mathrm{~mm}$ \\
Planarity of the horiz. surface of return yokes & $0.1 \mathrm{~mm}$ \\
\hline
\end{tabular}

Table 1. Main mechanical specifi cations for steel production and machining

\begin{tabular}{|l|c|c|c|}
\hline Element & Spec. & Slab steel & Yoke steel \\
\hline $\mathrm{C}$ & $<0.080$ & $0.08 \pm 0.01$ & $0.004 \pm 0.002$ \\
$\mathrm{P}$ & $<0.025$ & $0.011 \pm 0.003$ & $0.003 \pm 0.001$ \\
$\mathrm{~S}$ & $<0.010$ & $0.005 \pm 0.003$ & $0.005 \pm 0.002$ \\
$\mathrm{Mn}$ & & $1.24 \pm 0.13$ & $0.24 \pm 0.02$ \\
$\mathrm{Si}$ & & $0.20 \pm 0.03$ & $0.84 \pm 0.03$ \\
$\mathrm{~B}$ & $<0.0005$ & $<0.0001$ & $0.00016 \pm 0.00005$ \\
\hline
\end{tabular}

Table 2. Weight fraction of various elements according to specifi cations (column 2) and measured after the production of the slab steel (col.3) and return yoke steel (col.4). Units are in \% of weight.

the choice of the magnet steel is driven by severe mechanical constraints but its composition must be appropriate for magnetic applications. Each arm of the magnet is made up of 12 layers; each layer is obtained lining seven slabs $50 \times 1250 \times 8200 \mathrm{~mm}^{3}$. The mechanical specifications for the slab steel are summarized in table 1. In addition, to preserve the magnetic permeability, upper limits on the weight fractions for $\mathrm{C}, \mathrm{P}$ and $\mathrm{S}$ has been specified (see column 2 of table \&) both for the slab and return yoke steel. The chemical analysis was done by the steel producers on a batch (i.e. heat) to batch basis. In particular, the return yokes consist of six steel basements (1250 mm width) and two $625 \mathrm{~mm}$ half-basements; in this case each basement corresponds to a different batch. The slab and return yoke composition is summarized in table 2 , where the mean weight fractions and the corresponding RMS are indicated for various dopants.

The producers were requested to prepare small toroidal samples (outer diameter: $11.4 \mathrm{~cm}$ ) for each batch, which have been analyzed at CERN using a split-coil permeameter. Figure 2 shows the relative magnetic permeability as a function of $B$ for one typical sample of the slab steel, the return yoke steel and the steel used in 2001 for the construction of a prototype [4] of the magnet at the Frascati National Laboratories of INFN. It is evident that, due to the smaller Mn and C concentration the magnetic properties of the return yoke steel are superior compared with the slab steel. On average, the ratio of the relative permeabilities at $\mathrm{B}=1.55 \mathrm{~T}$ of return yoke steel and the slab steel is 1.67. On the other hand, the smallness of the $\mathrm{C}$ concentration in the return yoke makes difficult 


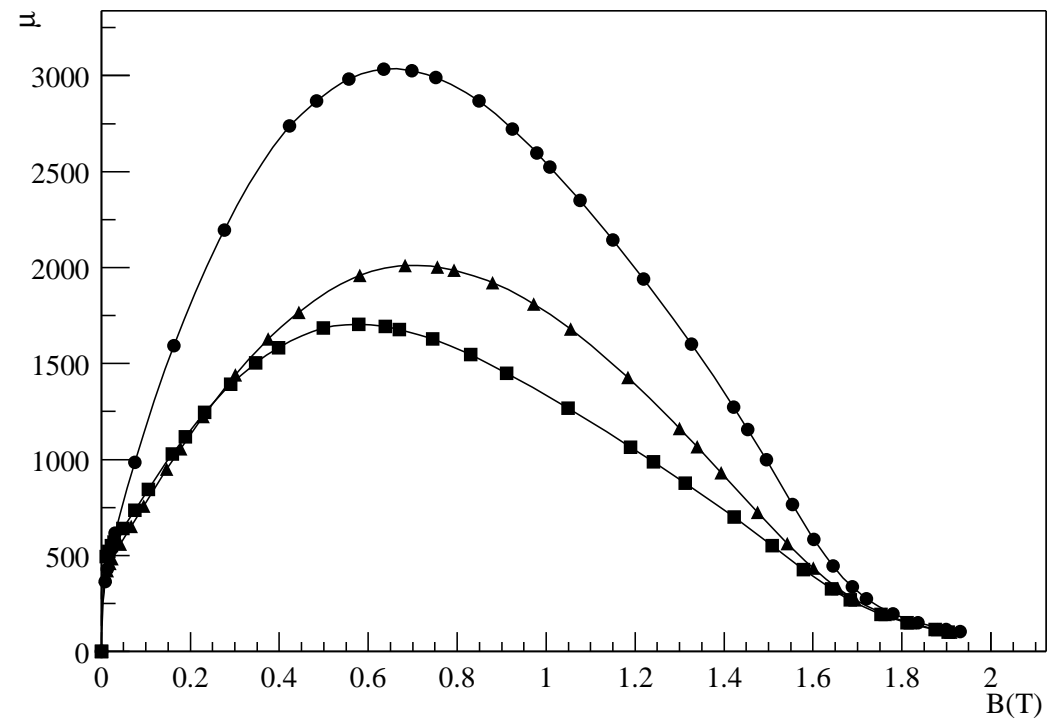

Figure 2. Relative permeability $\mu_{r}$ versus B for one sample of the slab steel (squares), the return yoke steel (circles) and the steel used for the construction of the prototype (triangles).

to keep under control local non-uniformities. Sample-to-sample variations are of the order of $12 \%$ $\left(\mu_{r}(1.55 T)=798 \pm 93\right)$, to be compared with the steel used for the slabs where variations can be kept below $1 \%: \mu_{r}(1.55 T)=476 \pm 6$. However, a better compromise between the mechanical and magnetic properties could have been obtained in the slabs by reducing the Mn content and optimizing the procedure for mass production (e.g. reheating). This is demonstrated by the performance of the prototype steel (triangles in figure 2), which retains appropriate mechanical properties but improves the magnetic response compared with the slab sample. Figure Đprovides information on the net effect on B due the choice of the slab steel. With respect to the prototype steel, $\mathrm{B}=1.55 \mathrm{~T}$ is obtained with a magnetomotive force $\sim 15 \%$ larger. The effect of the different steels has been evaluated from a finite-element analysis based on TOSCA (see section 4 and ref. [5]). At magnetomotive forces (m.m.f.) of the order of 64000 A.turns (i=1600 A, i.e. the nominal current at which the magnets have been operated during the 2006 run), the steel is close to saturation; here, the expected difference of the field in the arms with respect to an ideal magnet built with the prototype steel never exceeds $1.5 \%$. Note, however, that such m.m.f. is significantly larger $(+16 \%)$ than the one foreseen at the time of the prototype tests (55200 A.turns) [ [

\section{Electric characterization of the load}

Magnetomotive force to produce the $B$ field is provided by two DC power supplies, each located on the top of the magnet. They are single-quadrant $\mathrm{AC} \rightarrow \mathrm{DC}$ devices providing a maximum current of $1700 \mathrm{~A}$ and maximum voltage of $20 \mathrm{~V}$. The power supplies are connected to the driving coil wound in the return yokes of the magnet by means of short flexible cables. The coil is made up of $100 \times$ 
$20 \mathrm{~mm}^{2}$ copper (type Cu-ETP UNI 5649-71 ${ }^{1}$ ) bars. The segments are connected through bolts after polishing and gold-plating of the contact surface. Each coil is constituted by 20 turns in the upper return yoke connected in series to additional 20 turns in the bottom yoke. The two half are linked by vertical bars running along the arm. Rexilon supports provide spacing and insulation of the bars. Water heat exchangers are positioned between these supports and the bars while the vertical sections of the coil are surrounded by protective plates to avoid accidental contacts. More than 160 junctions have been made for each coil and the quality of such contacts was tested measuring the overall coil resistance during mounting. A 4-wire microohmmeter (Hewlett-Packard HP4284A) have been used to measure the bulk resistance of the coils. At $\mathrm{T}=20^{\circ}$ (average temperature of the coil) the values are $7.04 \pm 0.03(7.13 \pm 0.03) \mathrm{m} \Omega$, the errors being dominated by the uncertainty on the temperature of the various sectors of the coil. The theoretical value is $7.16 \mathrm{~m} \Omega$ and, therefore, contact resistance at junctions does not contribute significantly to the overall ohmic resistance of the load.

The voltage at the power supply is given by:

$$
V=R i+L \frac{d i}{d t}=\rho(T) \frac{l}{\sigma} i+\frac{d \Phi}{d t}=R i+N \frac{d}{d t} \int_{S} d S^{\prime} \mathbf{B}\left(S^{\prime}, t\right) \cdot \mathbf{n}
$$

$R, l$ and $\sigma$ being the resistance, length and transversal surface of the coil, respectively; $\rho(T)$ the copper resistivity and $\Phi$ the magnetic flux cut by the $\mathrm{N}$ turns along the iron surface S. Clearly, in the static limit $(t \rightarrow \infty$ and $d i / d t=0)$ the voltage is given by the ohmic component $R i$ and, therefore, it provides an indirect measurement of the average temperature of the coil. Figure 3 shows the ohmic voltage at the power supply as a function of time for $\mathrm{i}=1600 \mathrm{~A}$. The limit value $(13.4 \mathrm{~V})$ corresponds to an average temperature of $64^{\circ}$ in good agreement with the estimate coming from temperature probes.

In the transient regime, i.e. during the ramp-up of the power supply, an inductive component is also present. Calculation of $d \Phi / d t$ is trivial if the $B-H$ curve of the steel is known and build-up of eddy currents is neglected. Assuming a purely horizontal field $H(t)=n i=N i / \lambda$ nearly uniform along the surface $S$ ( $\lambda$ is the average length of the magnetic circuit), we get:

$$
\frac{d \Phi}{d t} \simeq N S \frac{d B}{d H} \frac{d H}{d i} \frac{d i}{d t}=N S \frac{d B}{d H} \cdot n \frac{d i}{d t}=L \frac{d i}{d t} .
$$

However, for large-core transformers as in the present case, it is well known [6] that the above formula strongly overestimates the instantaneous inductance $L(t)$ since the penetration of the field is slowed down by the currents arising into the core. Figure $\emptyset$ shows the inductive response $V(t)-$ $R \cdot i(t)$ as a function of time during the path $(b \rightarrow d)$ of the hysteresis cycle as defined in figure 5 . Here, the current is progressively increased at a speed of $13.8 \mathrm{~A} / \mathrm{s}$ until the nominal value of $1600 \mathrm{~A}$ is reached. It corresponds to $\mathrm{H}=2864 \mathrm{~A} / \mathrm{m}$ and a magnetomotive force of $64000 \mathrm{~A} \cdot$ turns.

The maximum instantaneous inductance of the magnets never exceed 0.4 Henry while

$$
\left.N S n \frac{d B}{d H}\right|_{\max } \simeq 4 \text { Henry }
$$

\footnotetext{
${ }^{1}$ Chemical composition: $\mathrm{Cu}+\mathrm{Ag}>99.9 \%, \mathrm{Bi}<0.001 \%, \mathrm{~Pb}<0.005 \%, \mathrm{O}_{2}<0.04 \%$. The nominal volume resistivity is $0.0175 \Omega \mathrm{mm}^{2} / \mathrm{m}$.
} 


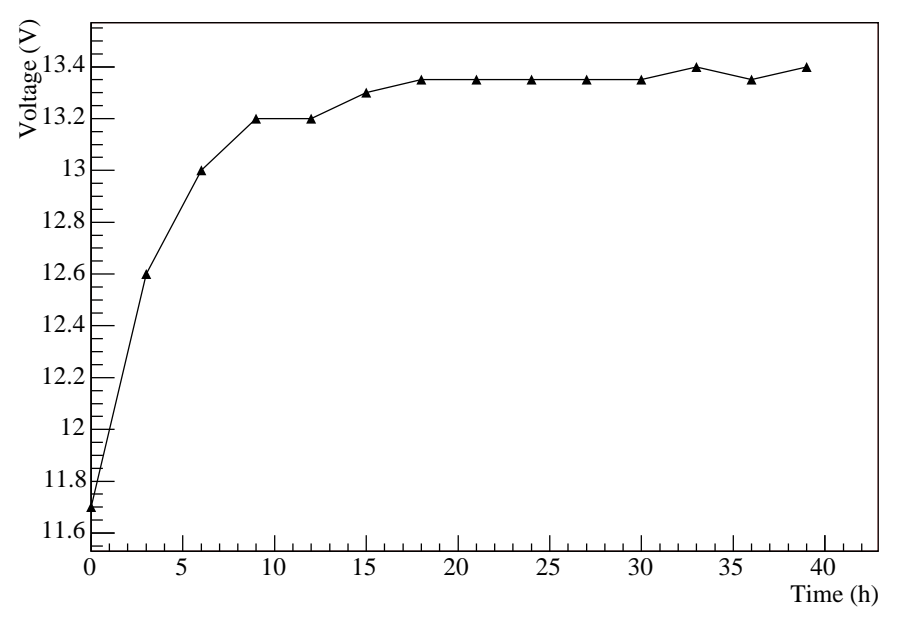

Figure 3. Voltage at the driving coil (i=1600 A) versus time (hours).

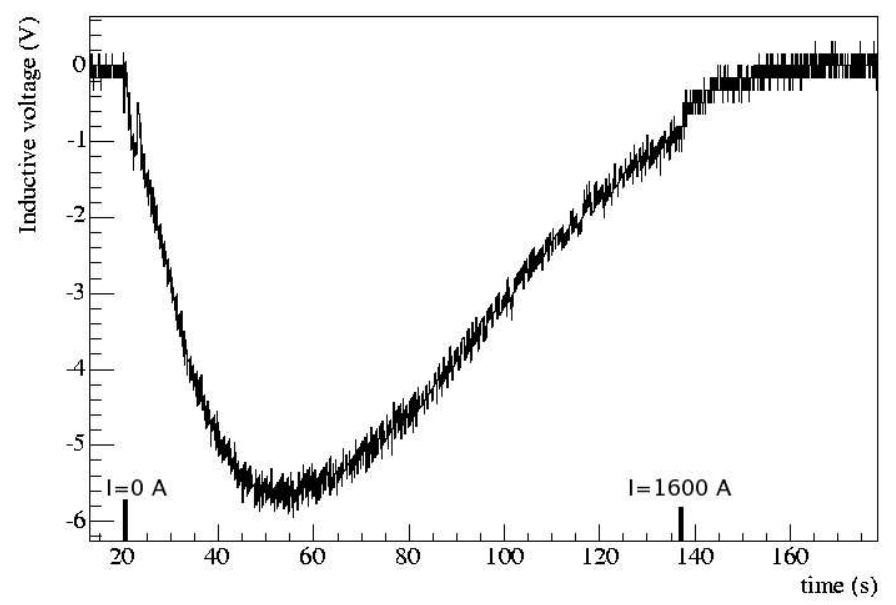

Figure 4. Inductive voltage $(V-R i)$ at the driving coil versus time during a ramp-up corresponding to path $b \rightarrow d$ of the hysteresis cycle as defi ned in fi gurø.

Even if the magnet is operated in DC, an estimate of the eddy current effect is needed for the design of the power supplies if a fast and stable ramp-up is desired. Moreover, the inductive response of the magnet during the increase of the driving current can be used to estimate the average magnetic field in the iron bulk. This procedure is described in the next section.

\section{Measurement of the magnetic field}

The geometry of the OPERA magnets has two advantages with respect e.g. to toroidal geometries: the magnetic field along the arms is much more uniform than in a toroid and it is essentially 1-dim., i.e. the components orthogonal to the vertical directions are nearly zero $\left(B \equiv|\mathbf{B}| \simeq B_{y}\right)$. Similarly to toroids, the magnetic circuit is confined within the steel (gapless) and therefore the measurement 


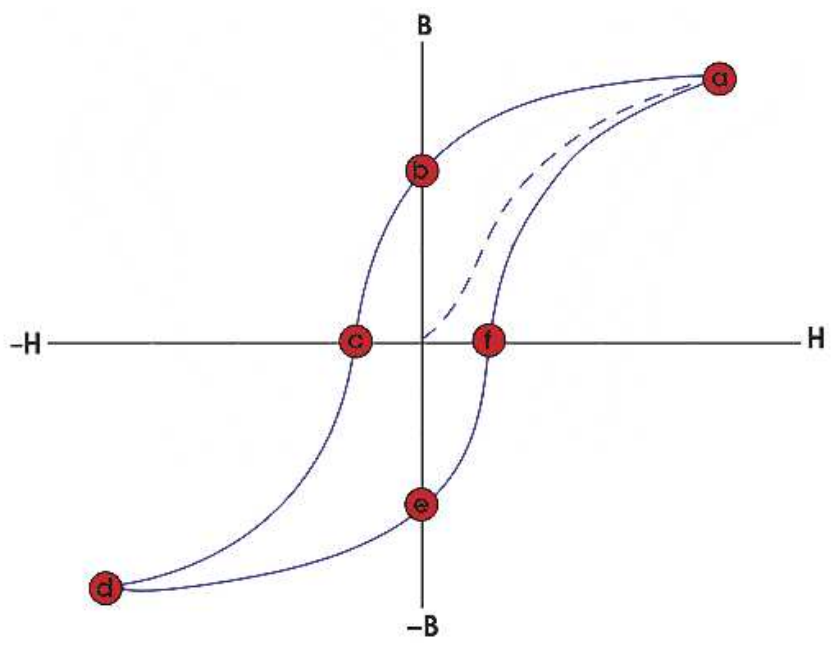

Figure 5. Paths used to measure the magnetic fi eld with the ramp-up technique.

of the fringe field in air provides only an indirect indication of $B$ in the arms. A direct measurement can be obtained from pick-up coils wound around the arms; integrating the induced voltage at these coils during ramp-up we have:

$$
\int_{0}^{T} d t V(t)=N_{p k} \frac{d \Phi}{d t} \simeq N_{p k} S \cdot[\langle B(t=T)\rangle-\langle B(t=0)\rangle]
$$

$S$ being the surface in iron cut by the coil and $\langle B(t)\rangle$ the average field in iron at time $t .^{2}$ Therefore, a full hysteresis cycle (see figure 5) provides two measurements of $|\mathbf{B}|$ at nominal current (1600 A). The current ramp of the power supplies can be set from $d i / d t=1$ to $13.8 \mathrm{~A} / \mathrm{s}$. Clearly, maximising the ramp-up time is useful because the induced voltage is proportional to $d i / d t$. A ramp-up (path $b \rightarrow d$ or $e \rightarrow a$ ) at maximum speed takes about $116 \mathrm{~s}$, however the ramp-down (path $d \rightarrow e$ and $a \rightarrow b$ ) takes much longer (several minutes). This is due to the fact that the power supplies employed for OPERA are single-quadrant, i.e. they cannot change continuously the sign of the voltage, ${ }^{3}$ hence, for sufficiently small i, $V=R i-L|d i / d t|<0$ and the constant ramp $d i / d t=-$ $13.8 \mathrm{~A} / \mathrm{s}$ cannot be sustained; in this case, more precise measurements of the field variation can be obtained ramping-up from point $e$ to $d$ or from $b$ to $a$ (these paths are not shown in figure 5 since $\Delta B_{e \rightarrow d}=\Delta B_{d \rightarrow e}$.

Figure 6 shows the distribution of 14 measurements (7 hysteresis cycles) done in one magnet with a pickup coil $\left(N_{p k}=32\right)$ wound around an arm at half-height. A single measurement has a precision of $\sim 2 \%$. At nominal current, the steel (see section 2) is close to saturation. The approach to saturation can be tested performing several hysteresis cycles at various $i_{\max }$. The results are plotted in figure 7 together with the theoretical expectation. The latter is drawn from a finite-element calculation in static regime. Modeling of the magnet has been done using the OPERA-3d [7] preprocessor and the corresponding Poisson equations are solved numerically by the TOSCA [7] code.

\footnotetext{
${ }^{2}$ In fact, the pickup coils cut also the vertical gaps where the RPC are located; in the present case, the corresponding flux can be safely neglected since $S_{\text {iron }}=(5 / 2) \cdot S_{R P C}$ and $B_{R P C} \ll B_{\text {iron }}$.

${ }^{3}$ The sign of the current, therefore, is obtained ramping down the power supply and inverting the load polarity through a motorized breaker.
} 


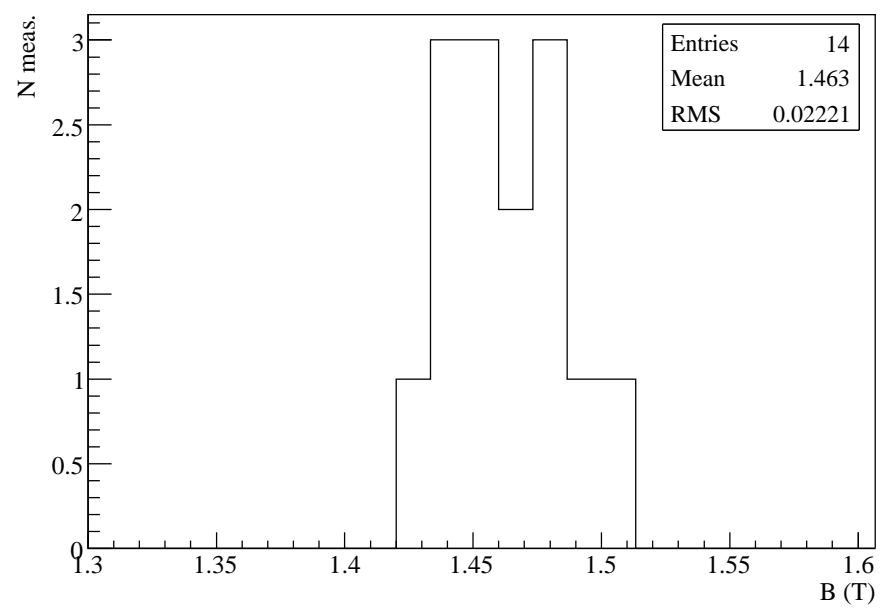

Figure 6. Distribution of the measurements of $B$ at $i=1600 \mathrm{~A}$.

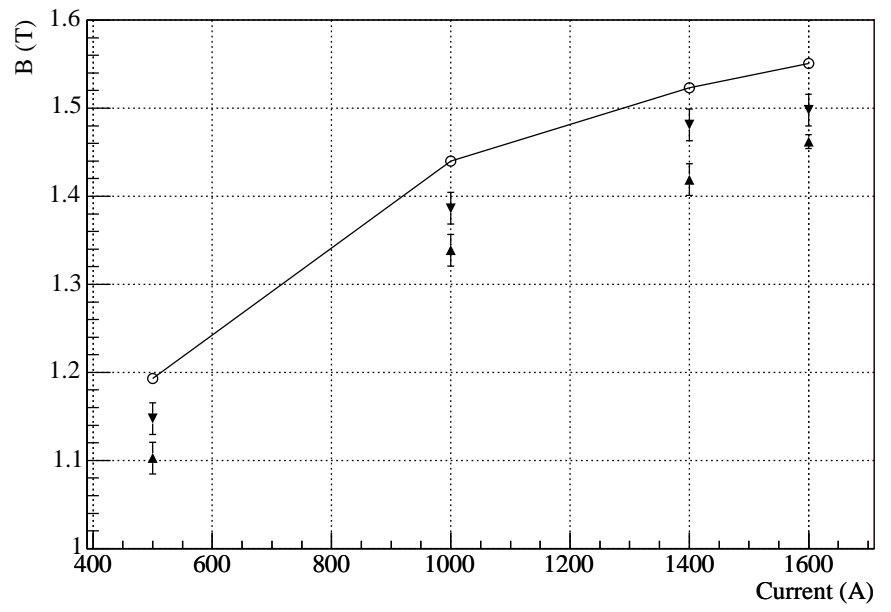

Figure 7. Field at the central pickup coil versus current. Empty dots represent the expectation from TOSCA; upward and downward full triangles are the measurements for magnets $n .1$ and 2 respectively.

The model includes the different magnetic response of the steels used for the return yokes and the slabs (section 2) but it assumes perfect mechanical contacts and neglects the effects of machining and lamination of the steel. A systematic deficit of flux (3-5\%) is clearly visible in the region where the half-height pickup coil is located; a similar effect has already been observed in the prototype of the magnet built at LNF-INFN in 2001 [2]. Note also the slightly better magnetic response of one magnet (n.2 in figure 7) with respect to the other (n.1). About the same deficit is observed at other locations of the pickup coils as shown in figure 8 .

The determination of the magnetic fringe field in air is relevant in OPERA due to the presence of multi-anode photomultipliers in the proximity $(\sim 1 \mathrm{~m})$ of the spectrometers. Indeed, the OPERA plastic scintillators are readout by 64-channel Hamamatsu H7546 PMT's. The fringe field perpendicular to the photocatode must be reduced below 5 Gauss and a dedicated iron shielding has been 


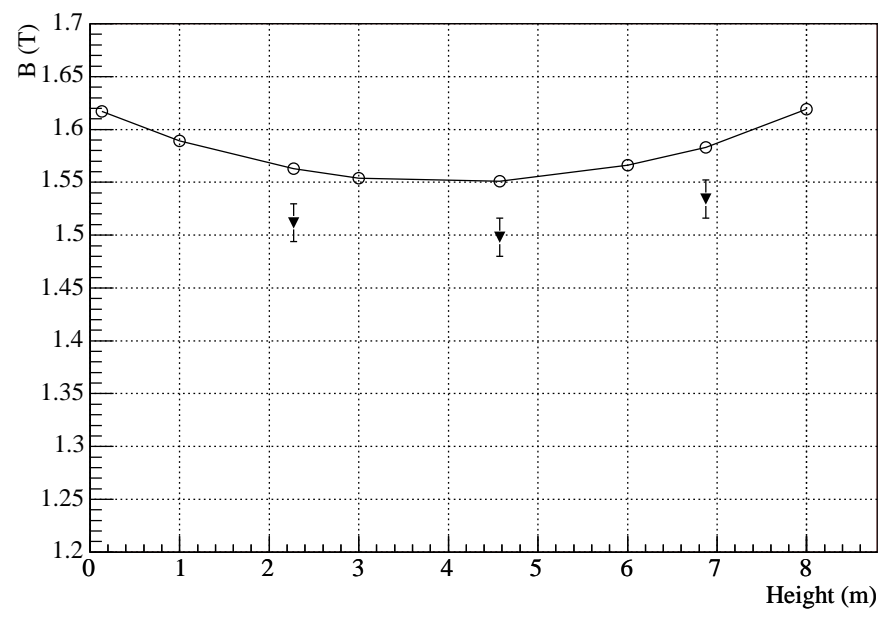

Figure 8. Measured fi eld (full triangles) at the pickup coils versus height. Empty dots show the expectation from TOSCA.

devised to suppress $B$ at this level [8]. The finite element calculation exploited before can be easily extended to provide a solid estimate of the field far from the coils even if numerical errors close to the RPC gaps or to the driving coils are quite large $(\sim 70 \%)$. Figure 9 shows the the expected field in air $(|\mathbf{B}|$ in Tesla) in the region near the photomultipliers. Measured points are also indicated: in particular, the second measurement from top indicates the observed value at the PMT closest to the border of the spectrometer. Excess with respect to simulation is observed only near the first layer of the magnet (third point from the top) and in between the slabs (fourth measurement from the top; here, the corresponding expectation is $\sim 60$ Gauss).

\section{Conclusions}

During 2006, a systematic characterization of the magnets for OPERA has been carried out. Relatively large differences among the steels of the return yokes and the vertical arms have been found, which are partially compensated operating the magnets near to saturation (i=1600 A). The minimum field at half height is $1.46 \pm 0.01 \mathrm{~T}$ while non-uniformities along the height do not exceed 3\%. A slight flux deficit w.r.t. simulation (3-5\%) is observed, likely due to the effect of non-ideal mechanical contacts and steel machining. Finally, the field in air in the proximity of the photomultipliers has been measured by means of Hall probes: this field never exceeds $28 \mathrm{G}$, in agreement with numerical expectations.

\section{Acknowledgments}

We wish to express our gratitude to A. Bergnoli, A. Garfagnini, L. Pellegrino and to the Technical Division of LNGS for their help in the operation of the magnets during this measurement campaign. A special thank to G. Corradi, who prepared the readout system for the pickup coils and to A. Mengucci and M. Spinetti for many useful suggestions. 


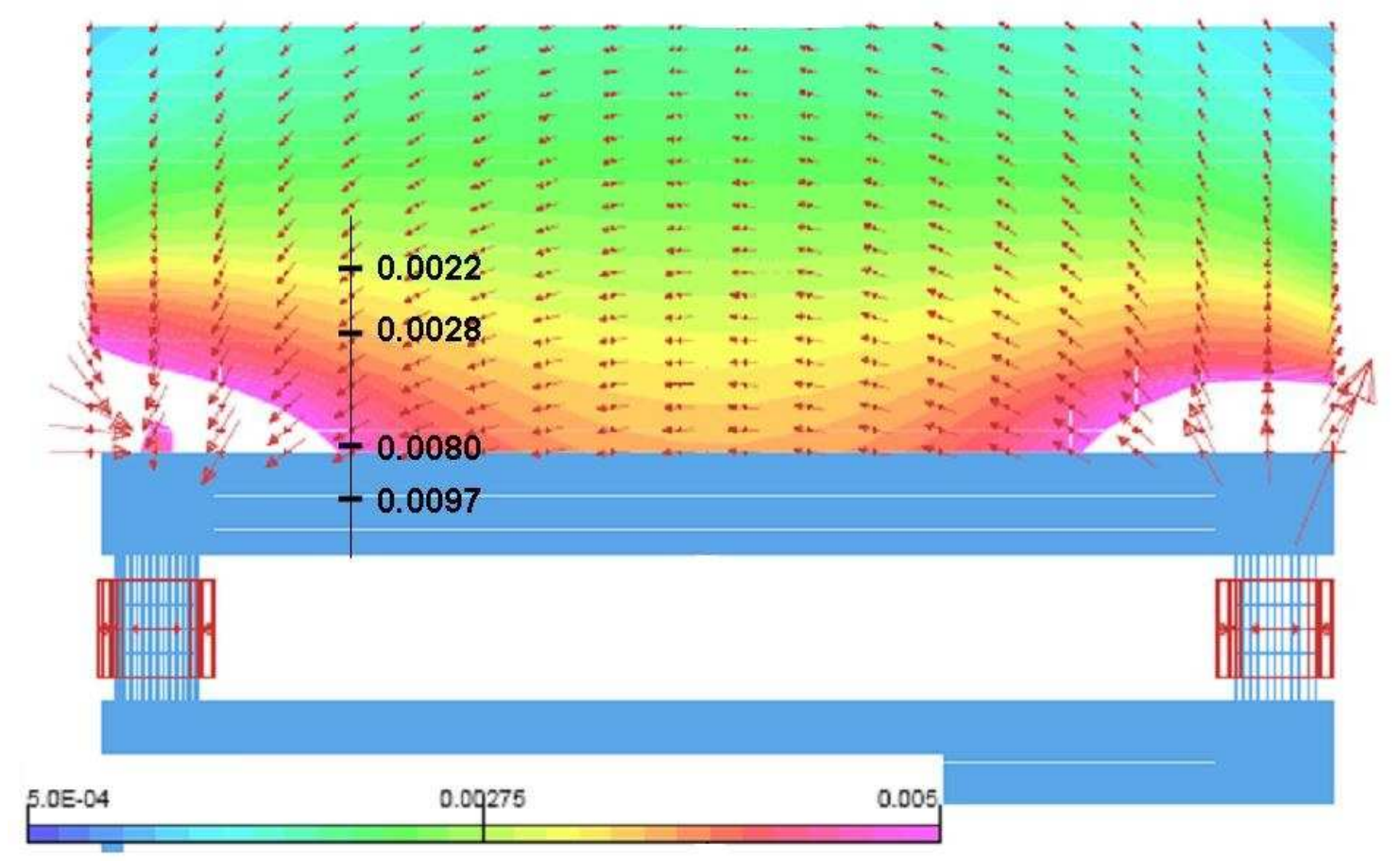

Figure 9. Expected fi eld in air $(|\mathbf{B}|$ in Tesla) in the region near the photomultipliers as computed by TOSCA. The vectors show the direction of the fi eld B. Measured points at two PMT's and near the magnet steel are also indicated (see text for details).

\section{References}

[1] M. Guler et al., An appearance experiment to search for $v_{\mu} \rightarrow v_{\tau}$ oscillations in the CNGS beam CERN/SPSC 2000-028, SPSC/P318, LNGS P25/2000, Jul. 2000.

[2] M. Ambrosio et al., The OPERA magnetic spectrometer, IEEE Trans. Nucl. Sci. 51 (2004) 975.

[3] R. Zimmermann et al., The precision tracker of the OPERA detector, Nucl. Instrum. Meth. A 555 (2005) 435 [Erratum ibid. A 557 (2006) 690].

[4] G. Di Iorio et al., Measurements of Magnetic Field in the Prototype of the OPERA Spectrometer, LNF-01/28, 2001.

[5] A. Cecchetti et al., Chemical and Magnetic Characterization of the Steels for the Opera Spectrometers, LNF-04/28, 2004.

[6] M. Incurvati and F. Terranova, Modeling skin effect in large magnetized iron detectors, Nucl. Instrum. Meth. A 500 (2003) 441 and references therein.

[7] OPERA-3d and TOSCA are products by Vector Field Ltd., Oxford, UK.

[8] M. Dracos et al., The OPERA Target Tracker Technical Design Report, available online at http://ireswww.in2p3.fr/ires/recherche/opera/general/TDR/target_tracker.htm 